\title{
Fruit and Vegetable Intake and Stomach Cancer among Male Adults: A Case-Control Study in Northern Viet Nam
}

\author{
Le Hong Phuoc ${ }^{1,2}$, Khanpaseuth Sengngam ${ }^{3}$, Toshio Ogawa ${ }^{1}$, Nlandu Roger \\ Ngatu $^{4}$, Shunya Ikeda ${ }^{1}$, Tran Hieu Hoc ${ }^{3}$, Pham Van Phu ${ }^{3}$, Dinh Thi Minh ${ }^{3}$, Le \\ Tran Ngoan ${ }^{4,5 *}$
}

\begin{abstract}
Objective: This study investigated the association between fruit and vegetable intake and stomach cancer, with considering the impacts of Helicobacter pylori (H. pylori) infection and tobacco smoking. Methods: A case-control study featuring 80 male incident stomach-cancer cases and 146 male controls was conducted in a general hospital in Viet Nam. A semi-quantitative food frequency and demographic lifestyle questionnaire were used; and venous blood samples were collected to determine $H$. pylori status by IgG ELISA. The respective associations between fruit and vegetable intake and stomach cancer were examined using unconditional logistic regression analysis with adjustments for possible cofactors. Results: Fruit intake and stomach cancer showed a weak inverse association when this became non-significant after adjusting for $H$. pylori infection $(\mathrm{OR}=0.50,95 \% \mathrm{CI}$ : $0.22-1.12$, $\mathrm{p}$ trend $=0.094)$. Stratifying by $H$. pylori status returned a negative trend for fruit intake and stomach cancer among $H$. pylori-negative participants $(\mathrm{OR}=$ $0.21,95 \% \mathrm{CI}$ : $0.06-0.69, \mathrm{p}$ trend $=0.010)$, but no significant interaction for $H$. pylori-positive participants $(\mathrm{OR}=0.76$, $95 \%$ CI: $0.21-2.68$, p trend $=0.670$ ). Vegetable intake and stomach cancer showed no association, regardless of $H$. pylori status. Compared to ever-smokers with low intake, never-smokers with high vegetable (OR $=0.25,95 \%$ CI: $0.06-0.95)$ and fruit intake ( $\mathrm{OR}=0.20,95 \% \mathrm{CI}$ : 0.06-0.65) showed the lowest odds of stomach cancer. Conclusions: Fruit, but not vegetable, intake showed a weak inverse association with stomach cancer. H. pylori infection and tobacco-smoking status may influence the protective effects of fruit and vegetable intake on stomach cancer.
\end{abstract}

Keywords: Stomach cancer- vegetable- fruit- Helicobacter pylori- tobacco smoking

Asian Pac J Cancer Prev, 21 (7), 2109-2115

\section{Introduction}

Globally, stomach cancer is the sixth most-diagnosed cancer, and the third most common cause of cancer death (Bray et al., 2018). The contributions of dietary factors to stomach cancer have been documented in numerous studies; in particular, high intake of salt and salt-preserved foods (Wang et al., 2009), pickles, cured and smoked foods (Ren et al., 2012), and processed meats (González et al., 2006) have been found to increase the risk of stomach cancer, while fruits and vegetables have been found to have protective factors in this regard (Correa, 2013). However, to date there has been no clear finding regarding the benefits of fruits and vegetables for stomach cancer (Lunet et al., 2005; Tsugane and Sasazuki, 2007; World Cancer Research Fund/American Institute for Cancer Research, 2018).
Chronic infection with $H$. pylori has also been found to be a carcinogen causing stomach cancer, and studies have suggested that fruit and vegetable intake modifies the effect of H. pylori infection on stomach cancer. Specifically, extracts from fruits and vegetables have been found to affect $H$. pylori infection in various methods, showing anti-H. pylori, anti-urease, anti-adhesive, and anti-invasive activities, as well as attenuating inflammation (Liu et al., 2018). Conversely, H. pylori infection may reduce the bioavailability of many anticarcinogenic vitamins and minerals widely found in fruits and vegetables (Zhang et al., 2002; Kim et al., 2005; Franceschi et al., 2014). The differing effects of fruits and vegetables on H. pylori-associated stomach cancer have been explored in some previous studies, but inconsistent results have been reported (Machida-Montani et al., 2004; Epplein et al., 2008; Wang et al., 2012; Wang et al., 2017). The above

${ }^{I}$ Graduate School of Public Health, International University of Health and Welfare, Narita city, Chiba Prefecture, Japan. ${ }^{2}$ Faculty of Public Health, University of Medicine and Pharmacy at Ho Chi Minh city, Ho Chi Minh city, Viet Nam. ${ }^{3}$ Hanoi Medical University, Ha Noi, Viet Nam. ${ }^{4}$ Department of Public Health, School of Medicine, International University of Health and Welfare, Narita city, Chiba prefecture, Japan. Institute of Research and Development, Duy Tan University, Da Nang city, Vietnam. *For Correspondence: lengoan@iuhw.ac.jp. Le Hong Phuoc, Khanpaseuth Sengngam have equal contribution in this study. 
findings suggest that $H$. pylori infection plays a crucial role as a confounder in the relationship between fruit and vegetable intake and stomach cancer. As with $H$. pylori infection, tobacco smoking is carcinogenic to humans and several studies have suggested that tobacco smoking status impacts the effect of fruits and vegetables on stomach cancer (Nouraie et al., 2005; Epplein et al., 2010).

The present study aimed to analyse the association between fruit and vegetable intake and stomach cancer among Vietnamese men, with adjustments for two wellknown risk factors: $H$. pylori infection and tobacco smoking.

\section{Materials and Methods}

\section{Study design and participants}

A hospital-based case-control study was conducted between January and December 2018 in a general hospital located in northern Viet Nam. The cases comprised Vietnamese men who had been newly diagnosed with stomach cancer through histopathology, and who had undergone surgery for cancer treatment. Controls included male Vietnamese patients who had no history of cancer (at any site), and who underwent surgery in the same department as the cases during the same period. Exclusion criteria included 1) being on a diet, and 2) having a severe health condition or other morbidity that affected dietary patterns. Based on the list of patients who had description of surgery each week during the study period, 672 patients were chosen and agreed to undergo a $H$. pylori test. Of these participants, 326 patients who were women or who were identified as having other cancers. Additionally, 120 were excluded because they had severe health conditions $(n=83)$, were duplicate patients $(n=14$; i.e., patients who were recruited on separate occasions by different interviewers), or were scheduled to undergo a $H$. pylori-examination the next time $(\mathrm{n}=23)$. A total of 226 eligible male participants were recruited for this analysis, comprising 80 incident stomach-cancer cases and 146 controls (Figure 1).

\section{Data collection}

Participants were interviewed by trained interviewers on the day immediately before his surgery using a validated semi-quantitative food frequency and demographic lifestyle questionnaire. This questionnaire obtained information regarding demographic characteristics, family history of cancer (within second-degree relatives); dietary factors, and lifestyle factors (including tobacco smoking and alcohol consumption). For tobacco smoking, all participants were categorised into three groups: current, ex, and non-smokers, respectively. Current smokers were individuals who had completely consumed one tobacco product (through a cigarette or waterpipe tobacco) within the last six months; individuals who were not current smokers but had consumed tobacco products in their lifetime were classified as ex-smokers. Current and ex-smokers were then classified as 'ever smokers', and non-smokers as 'never smokers'. In terms of alcohol consumption, participants were classified into the 'ever drinkers' category if they had consumed at least one serving of alcohol in one sitting within the last 12 months; all other individuals were allocated to the 'never drinkers' group.

Regarding dietary factors, all participants were asked, based on a validated semi-quantitative food frequency questionnaire (SQFFQ), about their average frequency of intake and the portion size over the last 12 months for 15 and 14 different types of vegetables and fruits, respectively. The SQFFQ used comprised 85 foods/recipes that cumulatively contribute up to $90 \%$ of the major nutrients; it was based on a database created from a 24hour recall study conducted on 300 general households in Northern Viet Nam in 2017 (Le et al., 2018). In the questionnaire, respondents ranked the fruits and vegetables in terms of frequency of intake using a seven-point scale (from 'seldom or never intake' to '1-3 times/day') and in terms of portion size using a three-point scale ('small', 'medium', and 'large'). Daily intake for each fruit and vegetable (in grams) was estimated by multiplying the frequencies, portion sizes $($ small $=0.8$, medium $=1$, large $=1.2$ ), and the average weights of the intake for the respective foods (minus the percentage of weight lost due to preliminary preparation, based on the Vietnamese food composition table, version 2007). Total fruit and vegetable intake (gram/day) were calculated by adding the amounts for all fruits and vegetables, respectively. Then, this total amount was divided into categories based on the nearest tertile; these tertiles were determined based on the distribution of intake among the controls.

To investigate the presence of $H$. pylori infection, 3-ml aliquots of venous blood (taken after overnight fasting) were obtained from each case and control. Anti- $H$. pylori serum IgG antibody titers were tested using an enzyme-linked immunosorbent assay (ELISA), based on the sandwich principle, through the use of a $H$. pylori IgG ELISA kit (RE56381; IBL International, Hamburg, Germany). H. pylori status was classified into three groups, based on a cut-off index (COI) provided by the manufacturer: negative $(\mathrm{COI}<0.8)$, equivocal $(0.8-1.2)$, and positive $(>1.2)$.

\section{Ethics}

The present study was approved by the ethics committees of Hanoi Medical University, Viet Nam and the International University of Health and Welfare, Japan. Additionally, written informed consent were obtained from all participants.

\section{Data analysis}

The collected data were analysed using Stata version 15.0 (Stata Corp, College Station, Texas). Unconditional logistic regression analysis, with adjustments for age, education level, family history of cancer, tobacco smoking, alcohol consumption, and $H$. pylori status was used to estimate the odds ratios (ORs) and 95\% confidence intervals $(95 \% \mathrm{CIs})$ for the association between fruit and vegetable intake and stomach cancer. To examine whether the relationship between fruit and vegetable intake and stomach cancer is affected by $H$. pylori status, all patients with equivocal results in the anti-H. pylori test were categorised into the negative group, creating two $H$. pylori 
serostatus groups: 'negative' and 'positive', respectively. Then, ORs and 95\%CIs for the relationship between each exposure were calculated for these two strata of $H$. pylori status. All p-values were two-sided, and $\mathrm{p} \leq 0.05$ (alpha value) was considered to indicate statistical significance.

\section{Results}

The mean ages of the cases and controls were $59.99 \pm 11.72$ and $54.54 \pm 11.64$ years, respectively (data not shown). Among the cases, 52.5\% and 36.3\% were current- and ex-smokers, respectively, while these figures among controls were $45.9 \%$ and $28.1 \%$, respectively. Among the 226 participants, $38.9 \%(\mathrm{n}=88)$, $18.6 \%(n=42)$, and $42.5 \%(n=96)$ were classified as negative, equivocal, and positive, respectively, regarding H. pylori status. The cases showed a greater percentage of $H$. pylori-positive members when compared to the controls $(50.0 \%$ versus $38.3 \%)$ (Table 1$)$. The estimated amount of intake (median [interquartile range], grams/ day) of the cases and controls were 85.72 [75.73-116.74] and 91.26 [72.58-122.65] for vegetables; and 67.12 [40.77-89.84] and 81.93 [59.65-101.62] for fruits, respectively (data not shown).

The results shown in Table 2 suggest an inverse association between fruit intake and stomach cancer. In Model 1, a significant negative trend between fruit intake and stomach cancer was indicated; this suggests that increased fruit intake is associated with lower odds of stomach cancer ( $p$ for trend $=0.046$ ). Compared to the first tertile for fruit intake, the second and third tertiles showed lower odds of stomach cancer $(\mathrm{OR}=0.72$, 95\%CI: $0.36-1.46$; and $\mathrm{OR}=0.45,95 \% \mathrm{CI}: 0.20-0.98$, respectively). However, in Model 2, which additionally adjusted for $H$. pylori status, the negative relationship between fruit intake and stomach cancer was no longer statistically associated; this was despite the fact that a decreasing trend was still observed across the three tertiles of fruit intake (tertile 3 versus tertile $1: \mathrm{OR}=0.50$, 95\%CI: $0.22-1.12$; p for trend $=0.094$ ). No association between vegetable intake and stomach cancer was found in either model.

After stratifying by $H$. pylori status (Table 3), a significant negative association between fruit intake and stomach cancer was observed among H. pylori-negative individuals ( $\mathrm{p}$ for trend $=0.010$ ), but no such statistically significant relationship was found among H. pylori-positive individuals ( $\mathrm{p}$ for trend $=0.670$ ). For H. pylori-negative participants, the odds of stomach cancer decreased across the fruit intake tertiles, with the ORs among the second and third tertiles being 0.49 (95\%CI: $0.17-1.36)$, and 0.21 (95\%CI: 0.06-0.69), respectively. No statistically significant association between vegetable intake and stomach cancer was observed, regardless of H. pylori status.

Regarding the joint effect of tobacco smoking and fruit and vegetable intake, compared to 'ever smokers' (i.e., current- and ex-smokers) and who had low vegetable intake, 'never smokers' who had a high vegetable intake showed the strongest inverse association between vegetable intake and stomach cancer $(\mathrm{OR}=0.25$, 95\%CI: 0.06-0.95; meanwhile, no statistically significant difference was observed for 'ever smokers' and high vegetable intake $(\mathrm{OR}=1.23,95 \% \mathrm{CI}$ : 0.63-2.40; Table 4). Similarly, compared to ever smokers with low fruit intake, never smokers with high fruit intake also showed the lowest odds of stomach cancer $(\mathrm{OR}=0.20,95 \% \mathrm{CI}$ : 0.06-0.65).

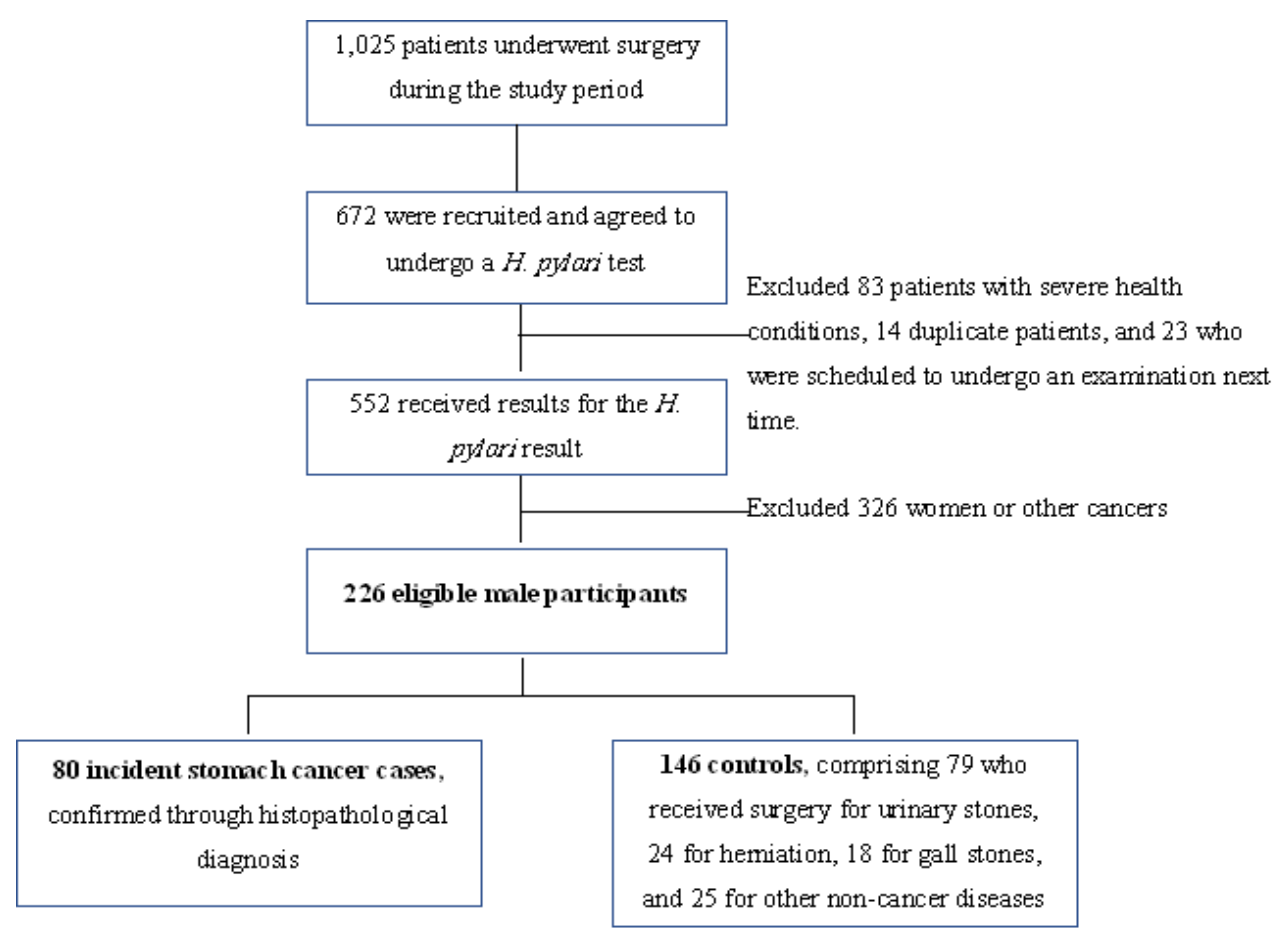

Figure 1. Participant Recruitment Process 
Table 1. Participants' Characteristics

\begin{tabular}{|c|c|c|c|}
\hline & $\begin{array}{c}\text { Cases } \\
(\mathrm{n}=80)\end{array}$ & $\begin{array}{l}\text { Controls } \\
(\mathrm{n}=146)\end{array}$ & $\mathrm{p}^{\mathrm{a}}$ \\
\hline & $\mathrm{N}(\%)$ & $\mathrm{N}(\%)$ & \\
\hline Age group (years) & & & 0.058 \\
\hline $20-29$ & $1(1.3)$ & $4(2.7)$ & \\
\hline $30-39$ & $3(3.7)$ & $12(8.2)$ & \\
\hline $40-49$ & $10(12.5)$ & $31(21.2)$ & \\
\hline $50-59$ & $24(30.0)$ & $53(36.3)$ & \\
\hline $60-69$ & $28(35.0)$ & $31(21.2)$ & \\
\hline$\geq 70$ & $14(17.5)$ & $15(10.3)$ & \\
\hline Education level & & & 0.231 \\
\hline Primary school & $16(20.0)$ & $19(13.0)$ & \\
\hline Secondary school & $36(45.0)$ & $64(43.8)$ & \\
\hline High school or higher & $28(35.0)$ & $63(43.2)$ & \\
\hline Family history of cancer & & & 0.238 \\
\hline Yes & $7(8.8)$ & $7(4.8)$ & \\
\hline No & $73(91.2)$ & $139(95.2)$ & \\
\hline Tobacco smoking & & & 0.009 \\
\hline Current & $42(52.5)$ & $67(45.9)$ & \\
\hline Ex & $29(36.3)$ & $41(28.1)$ & \\
\hline Never & $9(11.2)$ & $38(26.0)$ & \\
\hline Alcohol consumption & & & 0.034 \\
\hline Ever & $71(88.8)$ & $108(74.0)$ & \\
\hline Never & $9(11.2)$ & $38(26.0)$ & \\
\hline H. pylori status & & & 0.062 \\
\hline Negative & $25(31.3)$ & $63(43.2)$ & \\
\hline Equivocal & $15(18.7)$ & $27(18.5)$ & \\
\hline Positive & $40(50.0)$ & $56(38.3)$ & \\
\hline
\end{tabular}

\section{Discussion}

The present study findings suggest that there is a weak inverse association between fruit intake and stomach cancer, and that $H$. pylori infection potentially has a modifying effect on this association. After additional adjustment for $H$. pylori status, the negative relationship between fruit intake and stomach cancer became non-significant (Model 2). Moreover, subanalysis stratifying by $H$. pylori status (positive and negative) returned a significant association between fruit intake and stomach cancer among $H$. pylori-negative patients, but not among $H$. pylori-positive patients. Prior studies have also suggested that there is a weak inverse association between fruit intake and stomach cancer; several case-control studies (Lee et al., 2003; Lunet et al., 2007; Wang et al., 2012; Wang et al., 2017) have reported a significant negative association, while most cohort studies have not found a significant association (Larsson et al., 2006; Freedman et al., 2008; Steevens et al., 2011; Shimazu et al., 2014; World Cancer Research Fund/American Institute for Cancer Research, 2018). Regarding the joint effect of fruit intake and H. pylori infection on stomach cancer, the results of the present study are somewhat consistent with at least three comparable previous studies: one conducted in China (Wang et al., 2012), one in Japan (Machida-Montani et al., 2004), and a joint analysis conducted in China, Japan, and Korea (Wang et al., 2017). The first is a case-control study among a Chinese population (Wang et al., 2012) that reported a significant interaction between $H$. pylori infection and fruit intake in regard to non-gastric cancer ( $p$ for interaction $=0.011$ ). Although two later studies (the above-mentioned Japan-based study and the joint analysis) found no significant interaction in this regard they, nevertheless, indicated that $H$. pylori-positive individuals with low fruit intake have the greatest odds of stomach cancer (Machida-Montani et al., 2004; Wang et al., 2017). Reduced bioavailability and malabsorption of essential nutrients in fruits and vegetables may be a consequence of H. pylori infection; for example, H-pylori-positive individuals show decreased concentration of $\beta$-carotene in gastric juice, even when they have similar plasma levels as uninfected controls (Zhang et al., 2000). Moreover, the

Table 2. Adjusted Odds Ratios and 95\% Confidence Intervals for the Relationship between Vegetable Consumption, Fruit Consumption and Stomach Cancer

\begin{tabular}{lcccc}
\hline & $\begin{array}{c}\text { Cases }(\mathrm{n}=80) \\
\mathrm{N}(\%)\end{array}$ & $\begin{array}{c}\text { Controls }(\mathrm{n}=146) \\
\mathrm{N}(\%)\end{array}$ & $\begin{array}{c}\text { Model 1 } \\
\text { OR }(95 \% \mathrm{CI})^{\mathrm{a}}\end{array}$ & $\begin{array}{c}\text { Model } 2 \\
\text { OR }(95 \% \mathrm{CI})^{\mathrm{b}}\end{array}$ \\
\hline $\begin{array}{l}\text { Vegetable intake (grams/day) } \\
1(<78.55)\end{array}$ & $28(35.0)$ & $48(32.88)$ & 1.00 (reference) & $1.00($ reference $)$ \\
$2(78.55-108.83)$ & $28(35.0)$ & $47(32.19)$ & $1.40(0.67-2.93)$ & $1.28(0.60-2.73)$ \\
$3(>108.83)$ & $24(30.0)$ & $51(34.93)$ & $1.20(0.56-2.56)$ & $1.14(0.53-2.46)$ \\
p for trend & & & 0.637 & 0.736 \\
Fruit intake (grams/day) & $33(41.3)$ & $43(29.4)$ & 1.00 (reference) & $1.00($ reference $)$ \\
$1(<62.97)$ & $28(35.0)$ & $47(32.2)$ & $0.72(0.36-1.46)$ & $0.77(0.37-1.57)$ \\
$2(62.97-89.99)$ & $19(23.7)$ & $56(38.4)$ & $0.45(0.20-0.98)$ & $0.50(0.22-1.12)$ \\
$3(>89.99)$ & & & 0.046 & 0.094 \\
\hline
\end{tabular}

${ }^{a}$ Estimates were adjusted for age (years, continuous), education levels (primary school, secondary school, high school or higher, unknown), family history of cancer (yes, no), tobacco smoking (current, ex, never), alcohol consumption (ever, never), and fruit or vegetable intake (whenever one was not the main exposure); 'bstimates were adjusted for age (years, continuous), education levels (primary school, secondary school, high school or higher, unknown), family history of cancer (yes, no), tobacco smoking (current, ex, never), alcohol consumption (ever, never), fruit or vegetable intake (whenever one was not the main exposure), and H. pylori serostatus (negative, equivocal, positive). 
Table 3. Adjusted Odds Ratios and 95\% Confidence Intervals for the Relationship between Vegetable Consumption, Fruit Consumption, and Stomach Cancer in Terms of H. pylori Infection Status

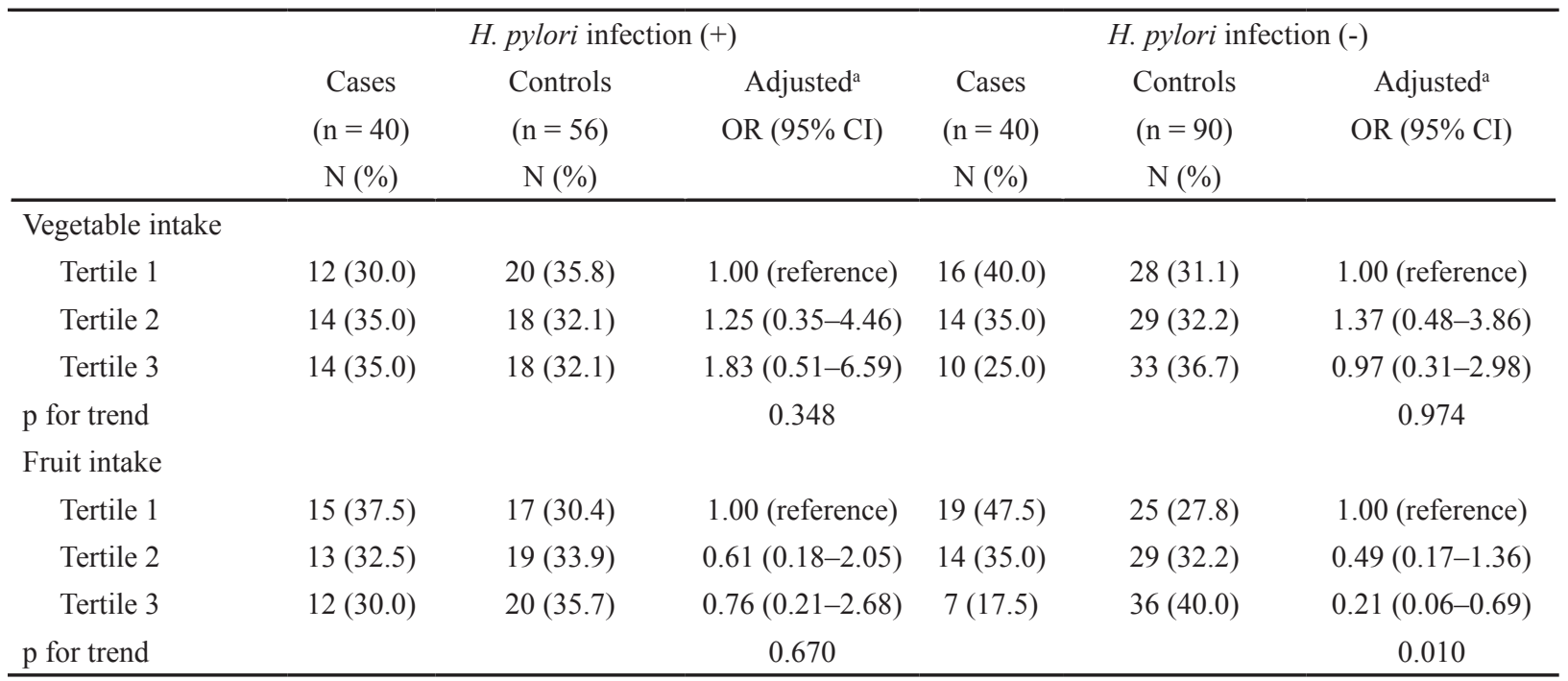

${ }^{a}$ Estimates were adjusted for age (years, continuous), education levels (primary school, secondary school, high school or higher, unknown), family history of cancer (yes, no), tobacco smoking (current, ex, never), fruit or vegetable intake (whenever one was not the main exposure).

bioavailability of other substances that can protect against stomach cancer, such as vitamin A, vitamin C, vitamin $\mathrm{E}$, and $\beta$-carotene, may also be reduced by $H$. pylori infection (Zhang et al., 2002; Kim et al., 2005; Franceschi et al., 2014). The above evidence suggests that $H$. pylori infection modulates the association between fruit intake and stomach cancer, weakening the protective effects of fruits for individuals with $H$. pylori infections.

The present findings indicate a null association between vegetable intake and stomach cancer, regardless of $H$. pylori status. There is, as yet, no consensus regarding the relationship between vegetable intake and stomach cancer. Two recent large cohorts (Gonzalez et al., 2012; Shimazu et al., 2014) have reported a marginally or non-significant association. Similarly, an updated report by the World Cancer Research Fund/American Institute for Cancer Research (World Cancer Research Fund/American Institute for Cancer Research, 2018) concluded that there is limited evidence (and no conclusion) regarding the association between the intake of vegetables and stomach cancer. This relationship, particularly in Asia and developing countries, should be investigated with caution, as a result of the high prevalence of $H$. pylori infections and the cooking methods used. The vegetable-consumption and cooking habits (i.e., boiling, frying, steaming) of Asian countries, including Viet Nam, may reduce the benefits of vegetable intake. In fact, a previous study suggested that there is a stronger inverse association between raw vegetables and cancers, particularly cancers of the upper gastrointestinal tract, when compared to cooked vegetables (Link and Potter, 2004). However, it should also be noted that epidemiological studies have suggested that foods, including fresh vegetables, play a role in the transmission of H. pylori (Quaglia and Dambrosio, 2018).

Regarding the influence of tobacco-smoking status on the effects of fruits and vegetables on stomach cancer, the

Table 4. Multivariate Odds Ratios and 95\% Confidence Intervals for the Joint Effects of Fruit and Vegetable Intake and Tobacco Smoking on Stomach Cancer

\begin{tabular}{llcccc}
\hline & & Cases $(\mathrm{n}=80)$ & $\begin{array}{c}\text { Controls }(\mathrm{n}=146) \\
\mathrm{N}\end{array}$ & $\begin{array}{c}\text { Unadjusted } \\
\text { OR }(95 \% \mathrm{CI})\end{array}$ & $\begin{array}{c}\text { Adjusted }^{\mathrm{b}} \\
\text { OR }(95 \% \mathrm{CI})\end{array}$ \\
\hline Tobacco smoking & Vegetable intake & & & & \\
Ever & $\leq 50 \%$ percentile & 37 & 56 & 1.00 (reference) & 1.00 (reference) \\
& $>50 \%$ percentile & 34 & 52 & $0.99(0.54-1.80)$ & $1.23(0.63-2.40)$ \\
Never & $\leq 50 \%$ percentile & 6 & 14 & $0.65(0.23-1.84)$ & $0.63(0.21-1.87)$ \\
& $>50 \%$ percentile & 3 & 24 & $0.19(0.05-0.67)$ & $0.25(0.06-0.95)$ \\
Tobacco smoking & Fruit intake & & & & \\
Ever & $\leq 50 \%$ percentile & 42 & 56 & $1.00($ reference $)$ & $1.00($ reference $)$ \\
& $>50 \%$ percentile & 29 & 52 & $0.74(0.41-1.36)$ & $0.81(0.42-1.57)$ \\
Never & $\leq 50 \%$ percentile & 5 & 10 & $0.67(0.21-2.10)$ & $0.63(0.19-2.15)$ \\
& $>50 \%$ percentile & 4 & 28 & $0.19(0.06-0.58)$ & $0.20(0.06-0.65)$ \\
\hline
\end{tabular}

a Fruit and vegetable intake were categorised into two: low intake $(<50 \%$ percentile) and high intake $(\geq 50 \%$ percentile) based on the distribution of intake among controls; 'bstimates were adjusted for age (years, continuous), education levels (primary school, secondary school, high school or higher, unknown), family history of cancer (yes, no), alcohol consumption (ever, never), fruit or vegetable intake (whenever one was not the main exposure), and H. pylori serostatus (negative, equivocal, positive) 
present study observed that high intake of fruits and/or vegetables is related to significantly lower odds of stomach cancer among never-smokers, but not among ever-smokers (compared to low-intake ever-smokers). Several studies have indicated that concentrations of $\beta$-carotene and vitamin $\mathrm{C}$ are significantly lower among smokers than non-smokers (Jarosz et al., 2000; Lykkesfeldt et al., 2000; Galan et al., 2005; Schleicher et al., 2009; Sugiura et al., 2009); moreover, in one study smokers showed lower plasma concentrations of these micronutrients than nonsmokers, even though both groups had consumed the same amount (Northrop-Clewes and Thurnham, 2007). Therefore, the present findings support the hypothesis that tobacco smoking is associated with a reduction in the stomach-cancer-associated benefit obtained from fruits and vegetables.

The present study has several limitations. First, the case-control design of this study is susceptible to reverse causation and recall bias. Information on food intake was collected for the period of 12 months preceding the interview; this relatively long duration may have made it difficult for participants to accurately report their fruit and vegetable intake habits. However, the SQFFQ was used in the present study, which has shown reasonable validity and reliability regarding capturing dietary information among the Vietnamese population when compared to 24-hour diet recall records (Le et al., 2018). Additionally, it is possible that the cases, as a result of their cancer diagnosis, changed their dietary habits to more frequent intake of fruits and vegetables; this may have led to an underestimation of the association between fruit and vegetables and stomach cancer. Therefore, a caution on the interpretation of the causal relationship between exposures and stomach cancer should be considered. Second, the recruitment of hospital-based controls may have contributed to bias in dietary assessment and might not reflect the dietary habits of the general population. Third, the small sample size used also is an important drawback that weakens the examined associations between the target exposures and stomach cancer. This may be the potential for observed non-significant associations including resulting in the false-negative for the relationship between fruit intake and stomach cancer (Model 2).

In conclusion, the present study suggested that there is a weak inverse association between fruit intake and stomach cancer, but no such association for vegetables. In addition, $H$. pylori infection and tobacco smoking appear to be important confounders for the relationship between fruit and vegetable intake and stomach cancer, attenuating the associated benefits provided by these foods.

\section{References}

Bray F, Ferlay J, Soerjomataram I, et al (2018). Global cancer statistics 2018: GLOBOCAN estimates of incidence and mortality worldwide for 36 cancers in 185 countries. $C A$ Cancer J Clin, 68, 394-424.

Correa P (2013). Gastric cancer: overview. Gastroenterol Clin North Am, 42, 211-7.

Epplein M, Nomura AMY, Hankin JH, et al (2008). Association of Helicobacter pylori infection and diet on the risk of gastric cancer: a case-control study in Hawaii. Cancer Causes Control, 19, 869-77.

Epplein M, Shu XO, Xiang YB, et al (2010). Fruit and vegetable consumption and risk of distal gastric cancer in the Shanghai Women's and Men's Health studies. Am J Epidemiol, 172, 397-406.

Franceschi F, Annalisa T, Teresa DR, et al (2014). Role of Helicobacter pylori infection on nutrition and metabolism. World J Gastroenterol, 20, 12809-17.

Freedman ND, Subar AF, Hollenbeck AR, et al (2008). Fruit and vegetable intake and gastric cancer risk in a large United States prospective cohort study. Cancer Causes Control, 19, 459-67.

Galan P, Viteri FE, Bertrais S, et al (2005). Serum concentrations of beta-carotene, vitamins $\mathrm{C}$ and $\mathrm{E}$, zinc and selenium are influenced by sex, age, diet, smoking status, alcohol consumption and corpulence in a general French adult population. Eur J Clin Nutr, 59, 1181-90.

González CA, Jakszyn P, Pera G, et al (2006). Meat intake and risk of stomach and esophageal adenocarcinoma within the European prospective investigation into cancer and nutrition (EPIC). J Nat Cancer Instit, 98, 345-54.

Gonzalez CA, Lujan-Barroso L, Bueno-de-Mesquita HB, et al (2012). Fruit and vegetable intake and the risk of gastric adenocarcinoma: a reanalysis of the European Prospective Investigation into Cancer and Nutrition (EPIC-EURGAST) study after a longer follow-up. Int J Cancer, 131, 2910-9.

Jarosz M, Dzieniszewski J, Dabrowska-Ufniarz E, et al (2000). Tobacco smoking and vitamin $\mathrm{C}$ concentration in gastric juice in healthy subjects and patients with Helicobacter pylori infection. Eur J Cancer Prev, 9, 423-8.

Kim HJ, Kim MK, Chang WK, et al (2005). Effect of nutrient intake and Helicobacter pylori infection on gastric cancer in Korea: a case-control study. Nutr Cancer, 52, 138-46.

Larsson SC, Bergkvist L, Wolk A (2006). Fruit and vegetable consumption and incidence of gastric cancer: a prospective study. Cancer Epidemiol Biomarkers Prev, 15, 1998-2001.

Le NT, Le HX, Pham PV, et al (2018). Reproducibility of a designed semi-quantitative food frequency questionnaire in general populations in the north Vietnam. Southeast Asian J Sci, 6, 191-200.

Lee SA, Kang D, Shim KN, et al (2003). Effect of diet and Helicobacter pylori infection to the risk of early gastric cancer. J Epidemiol, 13, 162-8.

Link LB, Potter JD (2004). Raw versus cooked vegetables and cancer risk. Cancer Epidemiol Biomarkers Prev, 13, 1422-35.

Liu Q, Meng X, Li Y, et al (2018). Natural products for the prevention and management of Helicobacter pylori infection. Comprehensive Rev Food Sci Food Safety, 17, 937-52.

Lunet N, Lacerda-Vieira A, Barros H (2005). Fruit and vegetables consumption and gastric cancer: a systematic review and meta-analysis of cohort studies. Nutr Cancer, 53, 1-10.

Lunet N, Valbuena C, Vieira AL, et al (2007). Fruit and vegetable consumption and gastric cancer by location and histological type: case-control and meta-analysis. Eur J Cancer Prev, 16, 312-27.

Lykkesfeldt J, Christen S, Wallock LM, et al (2000). Ascorbate is depleted by smoking and repleted by moderate supplementation: a study in male smokers and nonsmokers with matched dietary antioxidant intakes. Am J Clin Nutr, 71, 530-6.

Machida-Montani A, Sasazuki S, Inoue M, et al (2004). Association of Helicobacter pylori infection and environmental factors in non-cardia gastric cancer in Japan. 
Gastric Cancer, 7, 46-53.

Northrop-Clewes CA, Thurnham DI (2007). Monitoring micronutrients in cigarette smokers. Clin Chim Acta, 377, 14-38.

Nouraie M, Pietinen P, Kamangar F, et al (2005). Fruits, vegetables, and antioxidants and risk of gastric cancer among male smokers. Cancer Epidemiol Biomarkers Prev, 14, 2087-92.

Quaglia NC, Dambrosio A (2018). Helicobacter pylori: A foodborne pathogen?. World J Gastroenterol, 24, 3472-87.

Ren JS, Kamangar F, Forman D, et al (2012). Pickled food and risk of gastric cancer--a systematic review and meta-analysis of English and Chinese literature. Cancer Epidemiol Biomarkers Prev, 21, 905-15.

Schleicher RL, Carroll MD, Ford ES, et al (2009). Serum vitamin $\mathrm{C}$ and the prevalence of vitamin $\mathrm{C}$ deficiency in the United States: 2003-2004 National Health and Nutrition Examination Survey (NHANES). Am J Clin Nutr, 90, 1252-63.

Shimazu T, Wakai K, Tamakoshi A, et al (2014). Association of vegetable and fruit intake with gastric cancer risk among Japanese: a pooled analysis of four cohort studies. Ann Oncol, 25, 1228-33.

Steevens J, Schouten LJ, Goldbohm RA, et al (2011). Vegetables and fruits consumption and risk of esophageal and gastric cancer subtypes in the Netherlands Cohort Study. Int $J$ Cancer, 129, 2681-93.

Sugiura M, Nakamura M, Ogawa K, et al (2009). Synergistic interaction of cigarette smoking and alcohol drinking with serum carotenoid concentrations: findings from a middle-aged Japanese population. Br J Nutr, 102, 1211-9.

Tsugane S, Sasazuki S (2007). Diet and the risk of gastric cancer: review of epidemiological evidence. Gastric Cancer, 10, $75-83$.

Wang T, Cai H, Sasazuki S, et al (2017). Fruit and vegetable consumption, Helicobacter pylori antibodies, and gastric cancer risk: A pooled analysis of prospective studies in China, Japan, and Korea. Int J Cancer, 140, 591-9.

Wang X-Q, Terry P-D, Yan H (2009). Review of salt consumption and stomach cancer risk: epidemiological and biological evidence. World J Gastroenterol, 15, 2204-13.

Wang XQ, Yan H, Terry PD, et al (2012). Interaction between dietary factors and Helicobacter pylori infection in noncardia gastric cancer: a population-based case-control study in China. J Am Coll Nutr, 31, 375-84.

Zhang ZW, Abdullahi M, Farthing MJG (2002). Effect of physiological concentrations of vitamin $\mathrm{C}$ on gastric cancer cells and Helicobacter pylori. Gut, 50, 165-9.

Zhang ZW, Patchett SE, Perrett D, et al (2000). Gastric alpha-tocopherol and beta-carotene concentrations in association with Helicobacter pylori infection. Eur $J$ Gastroenterol Hepatol, 12, 497-503.

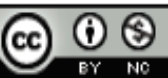

This work is licensed under a Creative Commons AttributionNon Commercial 4.0 International License. 